\title{
The Relativistic Addition of n (Scalar) Relative Velocities and a Short Possible Solution to Fermat's Last Theorem
}

\section{Lawrence $\mathbf{M}^{*}$}

Maldwyn Centre for Theoretical Physics, Ipswich, Suffolk, UK

\begin{abstract}
The paper shows how any number of (scalar) relative velocities due to external energies or forces can be added relativistically without the total velocity of the subject body or particle exceeding $\mathrm{c}$. The result is based on the product of fields rather than addition. It is also shown that Fermat's last theorem can be reduced from four variables to two and recast as the relativistic addition of real fractions of $\mathrm{c}$. The only rational solutions to the reduction correspond to powers of no higher than $\mathrm{N}=2$ in Fermat's conjecture.
\end{abstract}

Keywords: Fermat; Last theorem; Relativity; Addition; Velocity; Scalar; Product; Three-body problem; n-bodies; Many bodies

\section{Introduction - Addition of (Scalar) Velocities}

By considering the effects of gravity, charge or velocities as fields of any number of bodies on a single subject body or particle, and doing so in a way that includes all the acting bodies, the n-body or 3-body problems might become easier to solve. The route followed here is to look at scalar speeds (termed velocities for consistency) and parallel velocities based on the simplest accepted relativistic addition methodology for two velocities and extend it to any number. The result has an interesting link to Fermat's last theorem and it is shown that this link possibly enables a solution limited to $N=2$ and no higher [1-3].

\section{Significance and Objectives}

It is generally accepted that the relativistic addition of velocities $x$, $y$ as fractions of the speed of light $c$, considering only parallel velocities, follows the form:

$$
\text { Total velocity, } T_{r}=\frac{(x+y)}{(1+x y)}=T_{r}(2)
$$

where the meaning of $T_{r}$ and $T_{r}(2)$ will be explained below. This paper will show that this is only a specific example of a general form of treating scalar actions within any number of dimensions or of any number of interacting bodies, based on the interaction of, for example, $n$ fractional light speed velocities $i, j, k \ldots$ acting as the product of scalar fields thus:

$$
T_{G}(n)=\frac{\left\{\prod(1+i)-\prod(1-i)\right\}}{\left\{\prod(1+i)+\prod(1-i)\right\}}
$$

as will be explained below. It is then extended to suggest a short possible solution to Fermat's last theorem.

\section{Methodology}

This result can be obtained by starting simply and extending the treatment of $T_{r}$ to a third velocity, such that:

$$
\begin{aligned}
T_{r} & =\frac{\{x+(y+z) /(1+y z)\}}{\{1+x(y+z) /(1+y z)\}} \\
& =\frac{\{(x+y+z)+x y z\}}{\{1+(x y+x z+y z)\}}
\end{aligned}
$$

A new short method of describing these variables is now introduced. The summation of the variables on their own will be described as $\Sigma \Pi(1)$ meaning the summing of the product of variables of interaction value one, that is they do not interact with any other variables $[1,4]$. The summation of the cross products like $x y, x z$ and $y z$ will be described as $\Sigma \Pi(2)$, and here this would mean that $\Sigma \Pi(2)=(x y+x z+y z)$. The next in the series will be the triple interaction product $\Sigma \Pi(3)=x y z$. It is immaterial that in this example there is no actual summation because there is only one value of triple interaction, it standardises the use of this method. We now have the relativistic formula above as:

$$
T_{r}=T_{r}(3)=\frac{\left\{\sum \prod(1)+\sum \prod(3)\right\}}{\left\{1+\sum \prod(2)\right\}}
$$

What is found is that as the number of variables, here 3 , rises so the number of product parameters (here 1, $\Sigma \Pi(1), \Sigma \Pi(2)$ and $\Sigma \Pi(3)$ ) increases in line, with the actual numbers of cross components following a Fibbonnacci-like sequence. Starting at the left hand end of the denominator and then up to the left hand end of the numerator, alternating to next along thereafter, and keeping the value 1 in the $T_{r}(1)$ case, we get:

\section{$T_{r}(1)$ has 2 parameters with components 1,1 as $1, \Sigma \Pi(1)$}

$T_{r}(2)$ has 3 parameters with components $1,2,1$ as $1, \Sigma \Pi(1), \Sigma \Pi(2)$

$T_{r}(3)$ has 4 parameters with components $1,3,3,1$ as $1, \Sigma \Pi(1)$, $\Sigma \Pi(2), \Sigma \Pi(3)$

$T_{r}(4)$ has 5 parameters with components $1,4,6,4,1$ etc.

$T_{r}(5)$ has 6 parameters with components 1, 5, 10, 10, 51

$T_{r}(6)$ has 7 parameters with components $1,6,15,20,15,6,1$

and so on. Note that the sum of the numerator components is always equal to the sum of the denominator components, ensuring that the maximum value of any $T_{r}(n)$ is 1 .

Taking the $T_{r}(3)$ formula as a simple example, which can be

*Corresponding author: Lawrence M, Researcher, Maldwyn Centre for Theoretical Physics, Ipswich, Suffolk, UK, Tel: +223564565; E-mail: lawrence@maldwynphysics.org

Received September 21, 2017; Accepted October 31, 2017; Published November 03, 2017

Citation: Lawrence M (2017) The Relativistic Addition of n (Scalar) Relative Velocities and a Short Possible Solution to Fermat's Last Theorem. J Phys Math 8: 251. doi: 10.4172/2090-0902.1000251

Copyright: (c) 2017 Lawrence M. This is an open-access article distributed under the terms of the Creative Commons Attribution License, which permits unrestricted use, distribution, and reproduction in any medium, provided the original author and source are credited. 
generalised for $n$ velocities $i, j, k \ldots$, it can be rearranged to form:

$$
\begin{aligned}
\left\{1-T_{r}(3)\right\} & =\frac{\{(1-x)(1-y)(1-z)\}}{\{1+(x y+x z+y z)\}} \\
& =\frac{\left\{\prod(1: 3)(1-i)\right\}}{\{1+(x y+x z+y z)\}}
\end{aligned}
$$

where $\Pi(1: 3)(1-i)$ means the product of $(1-i)$ on each variable over three single variables $i, j, k[1,2]$. However, we can do the same for the denominator, where we get the result:

$$
\{1+(x y+x z+y z)\}=\prod(1: 3)(1+i)-\left\{\sum \prod(1)+\sum \prod(3)\right\}
$$

but it is also the case that,

$$
\prod(1: 3)(1+i)-\prod(1: 3)(1-i)=2\left\{\sum \prod(1)+\sum \prod(3)\right\}
$$

so that we now have:

$$
\left\{1-T_{r}(3)\right\}=\frac{2\left\{\prod(1: 3)(1-i)\right\}}{\left\{\prod(1: 3)(1+i)+\prod(1: 3)(1-i)\right\}}
$$

or

$$
\left\{1-T_{r}(3)\right\}=2 /\left[1+\left\{\prod(1: 3)(1+i) / \prod(1: 3)(1-i)\right\}\right]
$$

or

$$
T_{r}(3)=\frac{\prod(1: 3)(1+i)-\prod(1: 3)(1-i)}{\prod(1: 3)(1+i)+\prod(1: 3)(1-i)}
$$

which loses no strength when generalised to $n$ variables $i, j, k \ldots$ as:

$$
T_{G}(n)=\frac{\prod(1+i)-\prod(1-i)}{\prod(1+i)+\prod(1-i)}
$$

What this formula says is that the total (scalar) velocity is the difference between the products of the positive and negative scalar velocity fields present divided by their sum [5]. It also says that the value of $T_{G}(n)$ will always be less than or equal to 1 . A simple check for $n=2$ reveals:

$$
\begin{aligned}
& T_{G}(2)=\frac{\{(1+x)(1+y)-(1-x)(1-y)\}}{\{(1+x)(1+y)+(1-x)(1-y)\}} \\
& T_{G}(2)=\frac{\{(2 x+2 y)\}}{\{(2+2 x y)\}}=\frac{(x+y)}{(1+x y)}
\end{aligned}
$$

which is where we started.

\section{Summing Squares}

It is also clear that this can be extended to the square of total velocity or energy $T_{G}^{2}(n)$ and that the result is very different to that obtained by substituting $x^{2}$ for $x$.

This is shown by reverting to simple variables using $T_{G}(3)$ where the variables $x, y$ and $z$ are scalar velocities and by considering negative variables, we get:

$$
\begin{aligned}
& \left\{1-\left(-T_{G}(3)\right)\right\}=\frac{\{(1-(-x))(1-(-y))(1-(-z))\}}{\{1+((-x)(-y)+(-x)(-z)+(-y)(-z))\}} \\
& \left\{1+T_{G}(3)\right\}=\frac{\{(1+x)(1+y)(1+z)\}}{\{1+(x y+x z+y z)\}}
\end{aligned}
$$

From this, multiplying by $\left\{1 T_{G}(3)\right\}$ can be found:

$$
\left\{1-T_{G}^{2}(3)\right\}=\left\{\left(1-x^{2}\right)\left(1-y^{2}\right)\left(1-z^{2}\right)\right\} /\{1+(x y+x z+y z)\}^{2}
$$

If this is simplified by making $\mathrm{z}=0$,

$$
\left\{1-T_{G}^{2}(2)\right\}=\left\{\left(1-x^{2}\right)\left(1-y^{2}\right)\right\} /\{1+x y\}^{2}
$$

or

$$
T_{G}^{2}(2)=\left\{x^{2}+y^{2}+2 x y\right\} /\{1+x y\}^{2}
$$

or

$$
T_{G}^{2}(2)=\{x+y\}^{2} /\{1+x y\}^{2}
$$

which can be simplified to:

$$
T_{G}^{2}(2)=\left[\frac{\{x+y\}}{\{1+x y\}}\right]^{2}=\left\{T_{G}(2)\right\}^{2}
$$

and then compared with the 'usual' method of straight substitution of $x^{2}$ for $x, y^{2}$ for $y$ (and $z^{2}$ for $z$ ) in the first equation, which would instead give:

$$
T^{2}=\left\{x^{2}+y^{2}\right\} /\left\{1+x^{2} y^{2}\right\}
$$

It can be seen that there is a significant difference in both the numerators and denominators, even though at an extreme where $x=1$, both expressions result in $T_{2}=1$. The curve of each formula may start and end at the same points, but the curves are different between these ends $[6,7]$.

The new formula works in all dimensions, that is with any number of scalar velocities or energies acting. It is in the use for considering multiple overlapping fields or n-body problems that the utility of the $T_{G}$ (n) formula becomes apparent because rather than having to add fields, it is easier to multiply them and to understand what the interactions mean in terms of the cross components.

The meaning of the $T_{G}(n)$ formula is that the overall total scalar velocity of a body in multiple energy fields (travelling at multiple relative velocities, here in parallel but generalizable) is the difference between product of the sizes of those fields over and under the maximum field size ( 1 or velocity $c$ ) divided by the sum of those products.

If the addition method were extended to energy, then in a universe where energy can be considered to warp space, this is like considering any energy to be both positive and negative simultaneously, stretching space both 'upwards' and 'downwards' and comparing the difference between these stretches to the total energy involved in the stretching. Any variable that reaches 1 ensures that the total will also be 1 regardless of the size of any other variable. Thus space could have a maximum stretch of 1 in any 'direction' and not be ripped [8].

\section{Relativistic Factors}

There is also a distinct echo of the space-stretching in the formula for the relativistic factor $\gamma$, where:

$$
\frac{1}{\gamma^{2}}=\left(1-v^{2}\right)=(1-v)(1+v)
$$

being the product of both 'upward' and 'downward' velocity fields simultaneously. Here the value of $v$, the velocity as a fraction of light speed $c$, would be the outcome $T_{G}(n)$ when there were multiple parallel relative velocities to consider, so that:

$$
(1-v)(1+v)=\left\{1+T_{G}(n)\right\}\left\{1-T_{G}(n)\right\}
$$

Giving, 


$$
T_{G}^{2}(n)=1-\gamma(n)^{-2}=\frac{\left\{\prod(1+i)-\prod(1-i)\right\}^{2}}{\left\{\prod(1+i)+\prod(1-i)\right\}^{2}}
$$

And,

$$
\gamma(n)=\frac{\prod(1+i)-\prod(1-i)}{\sqrt{4 \prod\left(1-i^{2}\right)}}
$$

which continues to have the upward and downward products symmetrically included. And for $\gamma(1)$ we obtain, as expected,

$$
\gamma(1)=\frac{\{(1+i)+(1-i)\}}{\sqrt{4\left(1-i^{2}\right)}}=\frac{2}{\sqrt{4\left(1-i^{2}\right)}}=\frac{1}{\sqrt{1-i^{2}}}
$$

\section{Fermat's Last Theorem (Flt)}

To see where FLT fits it is necessary to recast his formula for real integers (using $N$ for the FLT power, rather than $n$, and $g$ rather than $c$ in the FLT equation, to avoid confusion)

$$
a^{N}+b^{N}=g^{N}
$$

into real fractions, limited to a maximum of 1 , by dividing each term by $g^{N}$ so that,

$$
\left\{\frac{a}{g}\right\}^{N}+\left\{\frac{b}{g}\right\}^{N}=1
$$

and then with $a<b<g$ and defining $b / g=\beta$ and $g / a=\gamma$, the equation looks like,

$$
\beta^{N}=1-\left\{\frac{1}{\gamma}\right\}^{N}
$$

or

$$
\begin{aligned}
& \beta^{N}=1-1 / \gamma^{N} \\
& \text { or } \\
& \frac{1}{\gamma^{N}}=1-\beta^{N}
\end{aligned}
$$

or

$$
\gamma^{N}=\frac{1}{1-\beta^{N}}
$$

This definition of $\gamma$ can be identified as the same type as in the previous section since it represents the same type of increase due to a single velocity or the inverse of the net field present. For $N=2$ we get:

$$
\beta^{2}=1-1 / \gamma^{2}
$$

which is the standard relativistic relationship, although more usually seen as:

$$
\gamma=\frac{1}{\sqrt{1-\beta^{2}}}
$$

and which was the result from the previous section for $\gamma$ (1)with $\beta=i$, confirming the definition of $\gamma$.

So when $N=2$ we have a well-known expression $[2,4]$. The question is whether it works for $N>2$. It does not matter for the moment that we are dealing with fractions, rather than integers, because we need to know whether there are any possible solutions for $N>2$ that would require unpacking the fractions. For $N=2$, the results are trivial, in that many integers in the original FLT equation can be converted into fractions that work correctly, such as $a=3, b=4, g=5$ giving $\gamma=5 / 3$ and $\beta=4 / 5$.
To find the constraints on the values of $\gamma$ and $\beta$, we need to look at the way that $\gamma$ was created above for velocity fields. The method was:

$$
\frac{1}{\gamma^{2}}=\left(1-v^{2}\right)=(1-v)(1+v)
$$

We note that there is no need to use any variable number $n$ because there is only one velocity, and that is $v=\beta$, so that we get:

$$
\frac{1}{\gamma^{2}}=(1-\beta)(1+\beta)
$$

We now raise that to the power of $N / 2$ and find that:

$$
\begin{aligned}
& \frac{1}{\gamma^{N}}=(1-\beta)^{N / 2}(1+\beta)^{N / 2} \\
& \text { or } \\
& \frac{1}{\gamma^{N}}=\left(1-\beta^{2}\right)^{N / 2}
\end{aligned}
$$

Now it must be the case, for FLT to be true at the same time as this equation is true, that:

$$
\frac{1}{\gamma^{N}}=\left(1-\beta^{2}\right)^{N / 2}=1-\beta^{N}
$$

This has reduced the number of variables from four to two. The equation:

$$
\left(1-\beta^{2}\right)^{N / 2}=1-\beta^{N}
$$

or more symmetrically:

$$
\left(1-\beta^{2}\right)^{N}=\left(1-\beta^{N}\right)^{2}
$$

has rational positive or zero solutions for $N>2$ of only $\beta=1,0$ as described below, although these solutions are not FLT solutions.

These two solutions imply $b=g, a=0$ or $b=0, a=g$.

To satisfy FLT in the new reduced two-variable form above for $N \geq$ 2 there need to be positive values for each of $a, b$ and $g$ and,

$$
\begin{aligned}
& \left(\frac{a}{g}\right),\left(\frac{b}{g}\right)<1 \\
& \left(\frac{a}{g}\right),\left(\frac{b}{g}\right)>0
\end{aligned}
$$

and

$$
\left(\frac{a}{g}\right),\left(\frac{b}{g}\right) \neq \text { complex or irrational numbers. }
$$

The solutions, other than $\beta=1,0,-1$, are complex and with increasing values of $N$ trend towards increasingly large negative or positive real and imaginary parts for both even and odd values of $N>2$.

What this means is that for $a, b, g$, as integers required by FLT, there are no rational fractions that are solutions for $N>2$. In terms of $a$ $b, g$, if any fractions are solutions that are irrational or complex, then at least one of $a, b, g$ could not be integers $[1,7,8]$.

So there is no requirement for the unpacking of the fractional values of $\beta$ or $\gamma$ and there are no rational fractions which satisfy the equations when $N>2$, and so no integers which satisfy the FLT in its original format.

\section{Conclusion}

The highest value of $N$ for which the FLT formulae work for all 
Citation: Lawrence M (2017) The Relativistic Addition of n (Scalar) Relative Velocities and a Short Possible Solution to Fermat's Last Theorem. J Phys Math 8: 251. doi: 10.4172/2090-0902.1000251

Page 4 of 4

possible values, in its reduced form, of $\beta$ and thus $a, b, g$, is $N=2$, and it is trivial to show that there are integer solutions that can be unpacked from the fractional values that satisfy the equations. This possible solution to FLT is suggested for discussion and it is accepted that it is not prepared in the usual mathematical method, but along a physics route.

\section{References}

1. Aldrovandi R, Pereira JG, Vu KH (2005) Gravity and the Quantum: Are they Reconcilable? Conference Proceeding of Quantum Theory: Reconsideration of Foundations-3. Vaxjo, University, Sweden.

2. Afshar SS, Flores E, McDonald KF, Knoesel E (2007) Paradox in Wave-Particle Duality. Found Phys 37: 295-305.
3. Bernhard C, Bessire B, Montina A, Pfaffhauser M, Stefanov AS (2014) Wolf Non-Locality of Experimental Qutrit Pairs. Journal of Physics A: Mathematical and Theoretical 47: 424013.

4. Ellis GFR (2007) Note on Varying Speed of Light Cosmologies. Gen Rel Grav 39: $511-520$.

5. Lawrence M (2016) How SI Units Hide the Equal Strength of Gravitation and Charge Fields. J Phys Math.

6. www.maldwynphysics.org.

7. Heisenberg W (1930) The Physical Principles of Quantum Theory. University of Chicago Press, USA.

8. Albert E (1936) Lens-Like Action of a Star by the Deviation of Light in the Gravitational Field. Science 84: 506-507. 